\title{
A Survey on Statistical Modeling and Machine Learning Approaches to Computer Assisted Medical Intervention: Intraoperative Anatomy Modeling and Optimization of Interventional Procedures
}

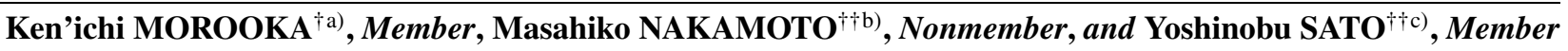

SUMMARY This paper reviews methods for computer assisted medical intervention using statistical models and machine learning technologies, which would be particularly useful for representing prior information of anatomical shape, motion, and deformation to extrapolate intraoperative sparse data as well as surgeons' expertise and pathology to optimize interventions. Firstly, we present a review of methods for recovery of static anatomical structures by only using intraoperative data without any preoperative patient-specific information. Then, methods for recovery of intraoperative motion and deformation are reviewed by combining intraoperative sparse data with preoperative patient-specific stationary data, which is followed by a survey of articles which incorporated biomechanics. Furthermore, the articles are reviewed which addressed the used of statistical models for optimization of interventions. Finally, we conclude the survey by describing the future perspective.

key words: statistical shape model, statistical deformation model, preoperating planning, surgical navigation, anatomical shape, organ motion, tissue deformation

\section{Introduction}

For the last two decades, a variety of computer assisted technologies for medical intervention have been developed. Rapid progress of medical imaging and position sensing technologies has accelerated the development. The earlydeveloped systems applied basic image processing and data integration methods to image and sensor data obtained from the patient who underwent an intervention. More recently, a large amount of the past patient datasets on anatomy, pathology, interventional planning, its execution, and outcomes have been accumulated, which could provide effective feedback information for the systems. Closed loop interventional medicine is a newly emerged paradigm which includes this feedback process [1]. Figure 1 shows its schematic diagram. In order to realize the paradigm, statistical modeling and machine learning approaches play a key

Manuscript received September 7, 2012.

Manuscript revised November 26, 2012.

${ }^{\dagger}$ The author is with the Graduate School of Information Science and Electrical Engineering, Kyushu University, Fukuoka-shi, 819-0395 Japan.

${ }^{1+}$ The authors are with the Graduate School of Medicine, Osaka University, Suita-shi, 565-0871 Japan.

a)E-mail: morooka@ait.kyushu-u.ac.jp

b) E-mail: nakamoto@image.med.osaka-u.ac.jp

c)E-mail: yoshi@image.med.osaka-u.ac.jp

DOI: 10.1587/transinf.E96.D.784 role in full utilization of these past patient datasets. Furthermore, sophisticated patient and interventional simulations using a large amount of computational resources are becoming possible [2], [3], which have been recently demonstrated to be effectively incorporated into intra-procedural assistance in a realtime manner by combining statistical modeling and machine learning approaches.

In this paper, we provide a survey of computer assisted medical intervention technologies using statistical modeling and machine learning approaches, which have also been shown to be useful for medical image segmentation [4] and medical image retrieval [5]. These approaches provide methods for modeling prior knowledge, such as statistical shape models [4], which can be utilized to realize less-invasive, highly accurate, and optimized interventions. That is, the prior knowledge is combined with sparse and incomplete imaging and sensing information as obtained during less-invasive procedures to estimate patient anatomy, organ motion, and tissue deformation [6] in a highly accurate manner. Furthermore, interventional procedures can be optimized by using the priors derived from a large amount of the patient datasets where the past intervention planning, execution, and its outcomes as well as patient anatomy are digitally recorded. This survey describes recent progress on these efforts.

In this paper, we do not deal with statistical models of surgical manipulation skill [7], [8] and surgical workflow [9]. Although these topics are important, the reason of their omission is due to the limitations of the authors' knowledge and specialty. In this survey, we focus on the narrower sense of computer assisted intervention, which is related to image and sensory information processing but not to medical robotics and surgical informatics.

The structures of the paper are as follows. In Sect. 2, intra-procedural anatomy modeling on shape and structures is described. In Sect. 3, modeling of organ motion and tissue deformation during interventions is described. In Sect. 4, incorporation of biomechanical simulations via statistical modeling and machine learning is described. In Sect. 5, statistical optimization of intervention procedures is described, and we summarize the paper in Sect. 6. 


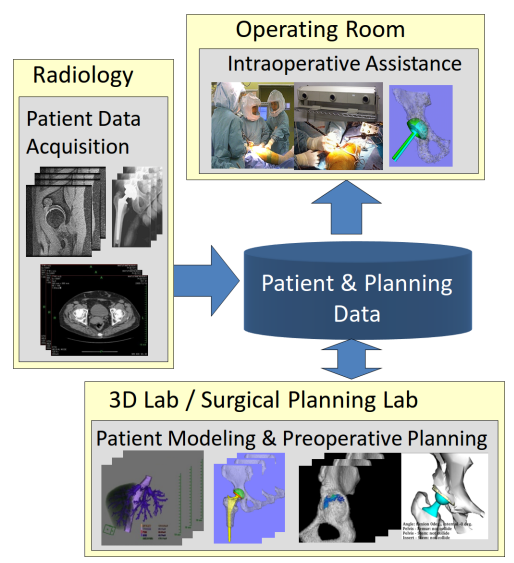

(a) Conventional computer assisted intervention

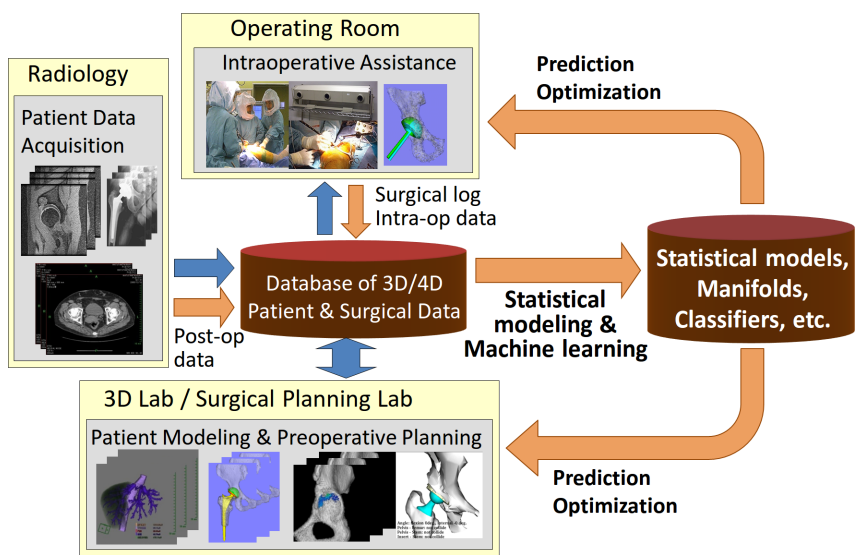

(b) Closed loop interventional medicine

Fig. 1 Schematic diagram of computer assisted intervention.

\section{Structure and Shape}

Statistical modeling and machine learning have been shown to be useful for predicting 3D anatomical shape and structures from sparse and incomplete image data as obtained during the intervention, especially in minimally-invasive procedures, even when any preoperative patient-specific 3D information is not available. In this section, we focus on modeling anatomical structures from datasets acquired using typical intraoperative modalities such as 3D digitizers, ultrasound (US) imaging, 2D X-ray fluoroscopy, and endoscopes without preoperative $3 \mathrm{D}$ datasets as acquired by $\mathrm{CT}$ and MR scanners. Although intraoperative CT and MR scans are becoming common, we did not include the methods using the intraoperative $\mathrm{CT}$ and MR data in this section because the methods for anatomical structure modeling using these datasets closely related to those using diagnostic CT and MR data, and thus the survey may not become wellfocused. Therefore, we only deal with the methods using typical intraoperative portable modalities.

So far, bones and urological organs are mainly targeted to estimate their anatomical coordinate systems, shapes, and density distributions. In clinical routine, 2D X-ray imaging is still a primary modality in orthopaedics for not only intraoperative but also preoperative imaging. US imaging plays a similar role in urology. In addition, these structures are typically assumed to be rigid or involve not large deformation. Therefore, intra-subject variability is not large in their shapes. Therefore, clinically sufficient accuracy is expected by combining statistical modeling and machine learning with intraoperative data without patient-specific preoperative data. Regarding urology applications, US imaging is mainly used and a comprehensive survey paper has already covered its intraoperative use [10]. Therefore, we focus on orthopedics in this paper. In the followings the details are described.

Orthopedic surgical navigation systems began to be clinically tested in mid-1990. These systems were based on rigid registration between preoperative $\mathrm{CT}$ bone surface

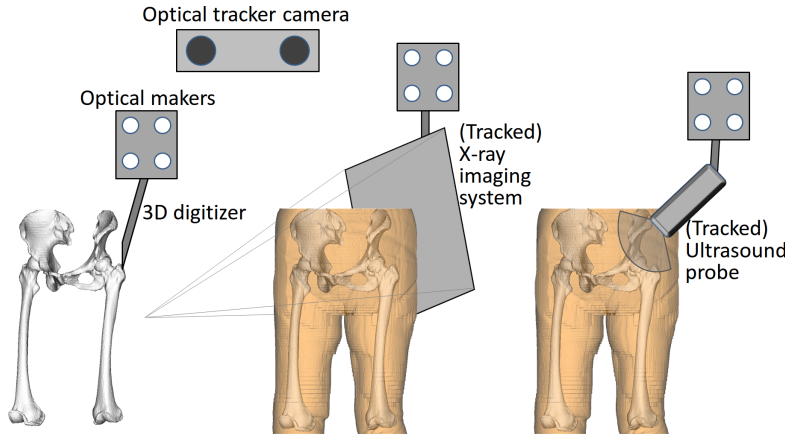

Fig. 2 Intraoperative sensing and imaging for orthopaedic applications of statistical modeling. Left: 3D digitizers need bone surface exposure to digitize it. Middle: X-ray imaging does not involve any incision to obtain bone shape information although X-ray irradiation is involved. Right: Ultrasound imaging is a non invasive method to obtain bone shape information although it field of view is narrow.

and intraoperative 3D positions on the actual bone surface acquired by a 3D digitizer. However, preoperative CT scans were not clinical routine in most orthopedic surgeries, especially in western countries. Eliminating the requirement of preoperative CT scans was essential for wide spread of surgical navigation in orthopedics. In order to intraoperatively recover 3D bone shapes, three modalities, 3D digitizer, 2D $\mathrm{X}$-ray, and US have been used in combination with statistical modeling approaches. The statistical shape model and its extension have been used for modeling bone shape as well as density distribution. The application targets have been mainly the knee, hip, and spine. Most of the methods are intended to be used for an intraoperative reconstruction of bone structures which are fed to computer-navigation systems. Figure 2 summarizes orthopedic applications using three main intraoperative sensing and imaging devices.

\subsection{D Digitizer}

One of the pioneering works on intraoperative 3D shape recovery of the bones without using preoperative patientspecific data was done by Fleute et al. [11], [12]. In order to 
realize surgical navigation without preoperative $\mathrm{CT}$, a statistical shape model (SSM), which is also known as an active shape model (ASM) [13], was applied to anterior cruciate ligament (ACL) surgery [11], [12]. In this application, the SSM of the distal femur was fitted to intraoperatively digitized 3D positions to reconstructed the bone shape. Ten femur datasets were used to construct the SSM of the distal femur, and the method was tested on the patients. The accuracy was evaluated by residual RMS (root mean square). This method was extended to intraoperatively reconstruct the proximal tibia as well as the distal femur and integrated into a surgical navigation system for total knee arthroplasty (TKA) [14]. This system provided a standard of so-called image-less navigation systems, which do not require any preoperative and intraoperative patient images.

\subsection{D X-Ray Imaging}

A portable 2D X-ray imaging system, which is called a "C-arm" system, is typically used in orthopaedic surgeries. A camera-calibrated and position-tracked C-arm system is a useful intraoperative tool for acquiring patient anatomy information. Recovery of 3D bone shapes and density distributions by combining $2 \mathrm{D} \mathrm{X}$-ray images and statistical models have been addressed. The problem is categorized in a class of nonrigid 2D/3D registration. Regarding technical aspects inherent in $2 \mathrm{D} / 3 \mathrm{D}$ registration such as similarity measures, matching features, and optimization methods, a recent survey paper [15] will be helpful. Here, we also describe methods developed for diagnostic 2D X-ray images which are suitable for application to intraoperative X-ray images.

Methods are classified into two categories. One deals with shape recovery, and the other both shape and density distribution. Some recent works of the former category do not require camera calibration of X-ray imaging. Regarding shape recovery, the initial trial of reconstruction from multiview X-ray images was done for the distal femur using SSMs by Fleute et al. [16] although the method was evaluated only by simulations, and the method was applied to the vertebrae [17]. Benameur et al. applied the SSM to the vertebra reconstruction from two-view images by introducing the regularization term defined as Mahalanobis distance from the average shape combined with the likelihood term of contour distance [18], [19], and further extended to deal with scoliotic vertebrae by extending the formulation to hierarchical SSMs [20], [21]. In these works, validations were done by dry bones, cadavers, and patients. The proximal femur and pelvis have also been targeted by SSM-based shape reconstruction from two-view (or multi-view) X-ray images [22]-[25]. Among them, Zheng et al. combined SSMs (Mahalanobis regularization) with the regularization using Mercer kernels [26] to further improve the reconstruction accuracy [24]. More recently, 3D shape reconstruction from a single uncalibrated X-ray image was developed for the pelvis [27], [28] and vertebra [29].

Regarding reconstruction of shape and density distribution, the research was initiated by Yao et al. in 2000 [30]. The series of researches targeted the pelvis and proximal femur[30]-[34]. A tetrahedral mesh density model was used for representing both shape and density distributions [31], and PCA was simultaneously applied to both of them to construct statistical shape and density model (SSDM) [32]. In the latest work, the femur in which bone cement was injected was tomographically reconstructed from incomplete projection datasets by using SSDM [33], [34].

\subsection{Ultrasound Imaging}

Since ultrasound (US) is portable and noninvasive modality, it is particularly suitable for intraoperative use. It has been utilized for soft tissue imaging but its bone imaging was not regarded as suitable for diagnostic purpose because US does not penetrate bone tissues. For surgical navigation purpose, however, a tracked and calibrated US can be used a non-invasive position data acquisition tool of bone surfaces instead of 3D digitizers and calibrated C-arm systems. The advantages of US are non-invasiveness and portability while the main disadvantage includes unclearness of bone boundaries and dependence of image quality on operators as well as patients. The initial validation of a US-based method was done for recovery of the cadaveric femur and pelvis using statistical deformation models (SDMs) by Chan et al. [35]. The SDM is regarded as a modified version of SSM and constructed from one reference pair of intensity and label images and a number of deformation fields obtained by nonrigid registration of each intensity image with the reference intensity image. SDM is regarded as an approximation of SSM. Considering accuracy and sparseness of US data, SDM is a practical choice because time-consuming preparation of a number of label images is not required. Through cadaveric validation, 3 or $4 \mathrm{~mm}$ accuracy was demonstrated. Its detailed descriptions are found in [36]. While the above works ([35], [36]) required a wide area of dense US scans which were time-consuming, Talib [37] and Rajamani [38] developed a method for recovery from sparse data and applied to the proximal femur, which was further extended to combine the smoothness constraint using Mercer kernels to improve the accuracy although experiment using US data was not conducted. [39]. More recently, SSM-based shape reconstruction using 3D US data was also applied to the lumbar spine for needle insertion purpose [40].

In the above, shape recovery of the whole 3D shape of the bone was addressed. Because an US probe is scanned manually, it is a time-consuming task even using a 3D probe to scan the probe so as to cover the whole shape. This point is a limitation for clinical application. In the total hip arthroplasty (THA) surgery, determining the anatomical coordinate system of the pelvis is required to define the orientation of the acetabular cup implant. Instead of whole shape recovery, a small number of small local ares are scanned for the coordinate system determination. These areas are typical anatomical landmarks (pubic symphysis, left and right an- 
terior superior iliac spines) for palpation in clinical routine. Therefore, the palpations are naturally followed by US scans in the surgical procedure. Foroughi et al. first addressed this problem [41], and attained clinically acceptable accuracy in dry bone and cadaver experiments. Schumann et al. applied patch-SSMs suitable for modeling multiple local areas for pelvis coordinate system determination in addition to sound speed compensation in SSM-fitting to US data [42], [43]. One problem of SSM-fitting of US data is how to determine the bone surface edges in the US images. Although sophisticated automatic edge detection methods from US images were studied [44], this problem is still under investigation. Ghanavati et al. developed a SSM-fitting method which does not require pre-segmentation of US images by direct local volume matching [45].

\section{Motion and Deformation}

Statistical approach is also applied to modeling of organ motion and deformation during therapy. Intraoperative imaging is commonly used to observe organ motion and deformation. 2D X-ray imaging and ultrasound imaging are conventional modalities for this purpose. However, 2D X-ray imaging has a risk of radiation exposure and it can acquire only $2 \mathrm{D}$ projection image. Ultrasound imaging can acquire even a 4D image recently, but it is not common and the field of view is not so large. These intraoperative images are sparse, limited field of view, and less quality, and thus they are sometimes insufficient to understand or reconstruct 3D organ motion. The statistical deformation model provides bases of parametric representation of organ motion. By combining the statistical deformation model with the intraoperative acquired patient-specific information, dynamic organ motion and deformation of the entire organ can be reconstructed. After reconstruction, by applying the reconstructed motion to the preoperative 3D image, you can see the organ motion with the higher quality preoperative 3D image. Davatzikos et al. proposed a framework of the statistical deformation model based on principal component analysis of displacement vector fields, which is extension of the statistical shape model [46].

In this section, we deal with the application of the statistical deformation model (SDM) to intervention. There are two main approaches to create a SDM. One is 4D image based approach, and another is biomechanical modeling based approach. In the 4D image based approach, a SDM is constructed from displacement vector fields extracted by inter-frame registration on the 4D image, in which actual organ deformation is recorded. The 4D image based approach is applied to the modeling of motion and deformation which can be caused easily at the time of image acquisition (e.g. respiratory motion). On the other hand, in cases where it is difficult to cause organ deformation beforehand (e.g. deformation caused by surgical operation), the biomechanical modeling approach is employed. This section focuses on the 4D image based approach and then the biomechanical analysis based approach is described in the next section. As already mentioned, the 4D image based approach is suitable for respiratory motion modeling. In the following subsections, we describe about SDM of respiratory motion of the lung and liver. The 4D image based SDM is also applied to the brain and the heart. Although statistical deformation models have been applied to long term changes of the anatomical structures in the brain [47]-[49] and the cardiac motion [50], [51], these studies aim to diagnostic purpose but not to intervention. Therefore, this paper does not describe them.

\subsection{Lung}

According to advance of radiation therapy technology, precise irradiation planning based on patient's 3D image and its execution are performed recently. Respiratory motion is the main factor of uncertainty in radiotherapy planning for the lung cancer, and then it reduces effectiveness of irradiation and causes larger irradiation margin. One approach to improve irradiation accuracy is estimation of cancer motion by using a prior knowledge of respiratory motion. The aim of the application of a SDM to respiratory motion is to predict near-future motion of the target organ from the previously obtained 4D image of respiratory motion. Because respiratory motion is repetitive and nearly cyclic, it is considered as a typical application of SDM. A statistical deformation model (SDM) reconstructed from extracted displacement vector fields from 4D image data are employed to represent variation of deformation caused by respiration. Zhang et al. developed a patient-specific respiratory motion model [52], [53]. In this method, the diaphragm position was incorporated into the motion model to express temporal correlations between the organ shape and diaphragm position. The motion model was applied to radiation treatment planning [52] and correction of motion artifacts in conebeam CT [53]. Liu et al. estimated a displacement vector field from the lung shape at arbitrary phase based on the assumption that there is linear correlation between the shape and the motion [54]. Ehrhardt et al. proposed a inter-patient mean motion model [55], [56]. They employed spirometry to estimate a scaling factor of the lung volume when instantiating a patient-specific motion from the model. As another application except radiation therapy, Barnett et al. reported application to respiratory gated PET scan to reduce artifacts in PET/CT [57]. Nakamoto et al. also proposed statistical modeling of bias error on registration caused by internal deformation that accompanies patient's position change to improve accuracy of registration for computer assisted surgery [58].

\subsection{Liver}

Since the liver is across the diaphragm from the lung, the liver also moves and deforms greatly with respiration. As well as the lung, the respiratory motion and deformation model of the liver is studied for assistance of the focal therapy of the liver. King et al. [59] and Blackall et al. [60] 
developed statistical respiration models, which were constructed from patient's own preoperative 4D CT image. King et al. also proposed a method for estimation of intraoperative liver shape by registering the statistical model to an intraoperative 2D ultrasound image. von Siebenthal et al. [61] developed an inter-subject statistical respiration model, which was constructed from 4D CT images of multiple subjects. In their method, a 3D surface model of each subject was divided into small cells based on anatomical features on the liver, and then it was registered to a reference shape model by using correspondence of the cells. Their method includes drift prediction. The organ drift is nonperiodic deformation and independent of the fitful breathing motion. Detection of the organ drift is difficult by using external sensors. Arnold et al. [62] developed a statistical exhalation drift model. They reported that estimation with the drift model showed significant improvement compared to estimation without the drift model. Lee et al. proposed dynamic shape instantiation, which can recover the entire 3D liver shape from the statistical model and ultrasound images acquired at limited imaging planes [63]. The relationships between the $3 \mathrm{D}$ surface model and very small subset of points on the liver contour appeared in an ultrasound image are trained by partial least squares regression (PLSR). The selection of suitable imaging planes results in the improvement of the accuracy and robustness of the shape estimation [64].

\section{Biomechanics}

Biomechanical modeling is the technique for accurately modeling the dynamic behaviors of living systems and their surrounding environment by basic mechanical properties. In the medical fields, biomechanical modeling is used to simulate the behaviors of target tissues whose experimental measurements are difficult or impossible. The simulation data provides significant benefits to diagnosis, surgical simulation and planning [65], [66]. Moreover, biomechanical modeling is used as constraints imposed on a non-rigid registration to avoid undesired registration. Owing to these advantages, biomechanical modeling has become a powerful tool in medical diagnosis and surgery.

Among the approaches for biomechanical modelings, the finite element method (FEM) can achieve a more physically realistic simulation for object behaviors with linear and nonlinear material properties. On the contrary, the FEMbased simulation is very time-consuming. Especially, the problem becomes serious for the surgical navigation and the interactive surgical simulator which requires real-time FE analysis. To solve this problem, the conventional methods using FEM [67], [68] introduce the assumptions of infinitesimal deformation and linear elasticity. There are many FEM approaches using the assumptions described in the survey paper [66]. Unlike such approaches, this section describes the biomechanical modeling combined with statical modeling and machine learning. One of the combination is to use simulated data by biomechanical modeling as sample

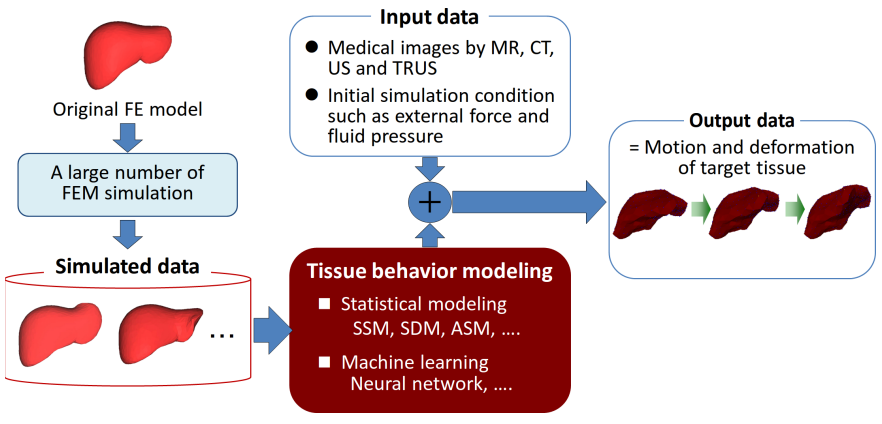

Fig. 3 Estimation of organ motion and deformation by combining biomechanical modeling with statistical modeling and machine learning approaches.

data for constructing statical models of motion and deformation of body organs including bone, brain and liver (Fig. 3). Such statistical model is called biomechanical based statistical model (BSM).

\subsection{Orthopedics}

The achievement of FE analysis with acceptable accuracy requires the accurate geometry and material property of target organs. As described in Sect.2.1, geometrical models of bones are accurately constructed from medical images. In addition, some works estimate bone material properties by the intensity of the bone CT images [69]. Owing to these reasons, since 1970's, the FE analysis has been a powerful tool in the filed of orthopedics surgery and diagnosis [70]. Querol et al. [69] designed a preoperative planning system of orthopaedic implant which predicts stress and strain data over bones by FE analysis. The system obtained the bone model by combining its statistical model with CT images. Gill et al. [71] developed a method for identifying a target spine by registering intra-operative spinal CT images to the virtual images derived from preoperative CT images. In the registration, FE analysis is incorporated into their registration algorithm to constrain the registration and accomplish anatomically acceptable alignment. Fitzpatrick et al. [72] developed a method for analysis implanted patellofemoral mechanics by combining a probabilistic and PCA approach. Their approach describes the relationship between input parameters and biomechanical output measurements. The input parameters include the alignments of patellar and femoral components while the output measurements are kinematics measurements and contact mechanics measurements.

\subsection{Neurosurgery}

One of the most widely used biomechanical modeling in neurosurgery is to estimate the deformation of brain structures caused by intraoperative brain shift [6]. The deformation leads the distortion of brain structures determined from the preoperative images. In this case, the direct use of the preoperative surgical planning is inappropriate for image- 
guided neurosurgery. The problem is solved by deforming the preoperative brain to intraoperative data based on BSM, which predicts the brain deformation. Dumpuri et al. [73] predicted intraoperative brain deformation by combining a statistical model of brain shift and sparse 3D points on the intraoperative brain surface measured by laser range scanner. Using the brain deformation model [74], their statistical model is constructed by a range of possible brain deformation simulation by changing boundary conditions.

Another application using biomechanical modeling is to construct patient-specific brain atlas for clinical diagnosis and preoperative planning. The patient atlas construction is based on the deformable registration of traditional atlas to patient images. Mohamed et al. formulated the finite element modeling of the brain structure deformation caused by the mass effect of bulk brain tumor growth [75], and further applied their FE modeling to the deformable registration method of the brain atlas to a patient brain with a bulk tumor [76].

\subsection{Other Soft Tissues}

BSM is introduced to the simulation for the deformation of soft tissues including skin and breast whose shapes are changed largely. The simulation is used for image-guided navigation system, and surgical training simulator. In the former, recent navigations combine BSM with 2D shape acquired by transrectal ultrasound (TRUS) to estimate intraoperative 3D shape of the tissues. Mohamed et al. [77] first developed the navigation which predicts intraoperative prostate shape using BSM and the position and pose of TRUS. Practically, the 3D intraoperative shape is constructed by minimizing the difference between 2D shape derived from the ultrasound and 3D shape estimated by BSM. Further, Hu et al. [78]-[80] improved the accuracy of the shape estimation by incorporating two components into the approach in [77]. One is to build BSM from a large number of FEM simulations by using FE models of the prostate gland, the pelvis, and the bladder. The simulation is made by changing the material properties of the models and the boundary conditions including the position and size of the pelvis relative to the prostate. The other is to perform a robust registration of the shape estimated by the SMM to TRUS images. The method in [79] was applied to five patients' TRUS images while the method in [80] was verified by eight patients' MR images. Moreover, Hu et al. [78] developed a efficent SSM constrution by simplifying the complex biomechanical modeling without loss of the estimation accuracy. Tanner et al. [81] developed a method for registering multimodal images by using the BSM which captures the motion and deformation of a breast compressed by two plates in the mammography. Their method was validated by using Xray-mammograms, and CT and MR images of a patient.

The shape estimation using BSM requires iterative computations. To solve the problem, Lee et al. [63] introduced partial least squares regression (PLSR) which describes the relationship between 2D liver contours as input data and 3D liver shape as output data. The various shape models are generated by non-linear FEM. Since the operations in PLSR are matrix multiplication, the efficient shape estimation is achieved.

Biomechanical modeling provides a powerful tool to construct surgical training simulator for the treatment of soft tissues. In the simulator construction, naturally, the real-time estimation of tissue deformation sequence is required. Moreover, since input data used in the estimation depends on the design of the simulator, it is necessary to model the relationship between the input data and its corresponding deformation. He et al. [82], [83] developed a BSM-based method for efficiently estimating bone-related deformation [83] and material parameter of soft tissue [82] by dividing BSM into two parts: the boundary part related to the bone, and the non-boundary part. Sieera et al. [84] estimated the uterine deformation in real-time which is caused by the pressure of the distension fluid during hydrometra. The real-time estimation is achieved by the linear combination of the uterine deformation precomputed offline by FEM. Further, Harders et al. [85] extended their method to treat various uterine shapes.

A neural network is useful to model the relationship between arbitrary input parameters and its corresponding outputs including 3D deformation shapes. To learn the relationship, the network is trained by using a large number of training data simulated by FEM. Kahalaji et al. [86], [87] combined BSM with the network which outputs the 2D shape deformation of prostate gland and 2D stress field of the shape when the positions of the landmark on the prostate contour and the material property assigned to the FE model are give. Morooka et al. applied the neural network to estimate the liver deformation by forces caused by surgical instruments [88], and further extended their method to apply the stomach deformation with large deformation [89]. The network training using a large dataset may not be converged. To solve the problem, the large dataset is partitioned into several small sub-datasets, and the simulator is constructed by multiple networks, each of which is trained by using its corresponding sub-dataset. When external forces caused by surgical instruments are used as input data of the simulator, the approximation or the imitation of the FEM simulation may be achieved by using mass-spring model [90] and FreeForm Deformation (FFD) [91]. In this case, the parameters in the mass-spring and FFD models are adjusted by fitting the deformations of these models to the FEM simulations used as training data. Mass-spring model and FFD may allow real-time deformation because of their computationally efficient. However, the deformation using these methods is inaccurate compared with the original FEM.

\section{Optimizing Intervention}

Computer assisted intervention mainly consists of two components. One is intraoperative assistance and the other preoperative planning. In the previous sections, issues of 
estimating anatomical shape, motion, and deformation for intraoperative assistance have been addressed. In this section, statistical modeling and machine learning approaches to preoperative planning are addressed. A lot of software systems for 3D interactive simulation and planning of interventional procedures have been developed, which are mainly based on technologies of visualization, virtual reality, image analysis, and so on. Although these systems provide intuitive, objective, and quantitative information to the surgeons, the optimization of interventional planning is performed by the surgeons. By introducing statistical modeling and machine learning, the systems are expected to provide the optimized planning incorporating a number of criteria which are in trade-off relations or may not be explicitly recognized even by experienced surgeons. Statistical approaches provide opportunities of fully utilizing the database of past surgical cases, in which information on patient anatomy and pathology as well as surgeons' expertise are embedded. In the following, recent achievements of these approaches are described by focusing on particular clinical purposes.

\subsection{Biopsy Planning}

\subsubsection{Prostate Cancer Biopsy}

Intraoperative assistance ensures accurate positioning of preoperatively planned biopsy locations. This assistance will be obviously useful for accurate biopsy. From the clinical viewpoint, however, the effectiveness of biopsy may be evaluated by the correct diagnosis ratio of the target disease by biopsy rather than positional accuracy of biopsy locations. For the biopsy of prostate cancer, Shen et al. proposed a statistical modeling approach to solve a problem which is more directly related to improving the effectiveness of biopsy from the clinical point of view [92], [93]. The problem is as follows: Given the prostate shape of the patient, find a fixed number of optimal biopsy locations minimizing the probability to miss the cancer tissue if the patient has it. In order to solve the problem, the probabilistic atlas was introduced which represents the prior probability map of cancer existence. Pathological images were used to obtain the distributions of cancer existence, which are typically unobservable or only ambiguously imaged in the clinical images.

Figure 4 shows the schematic diagram for derivation of the optimal prostate biopsy planning. The processes of constructing the probabilistic atlas are as follows. (1) Training datasets of labeled patient 3D images are prepared each of which includes the prostate region and cancer regions. The training datasets are obtained from pathological images. (2) The cancer regions in each training dataset are mapped to the standardized prostate space (defined as its average shape) so that the prostate region is registered to the average shape. (3) All the mapped binary cancer regions are averaged to construct the probabilistic atlas, which represents the existence probability of the cancer tissue at each voxel. Shen et al. determined the optimal biopsy location

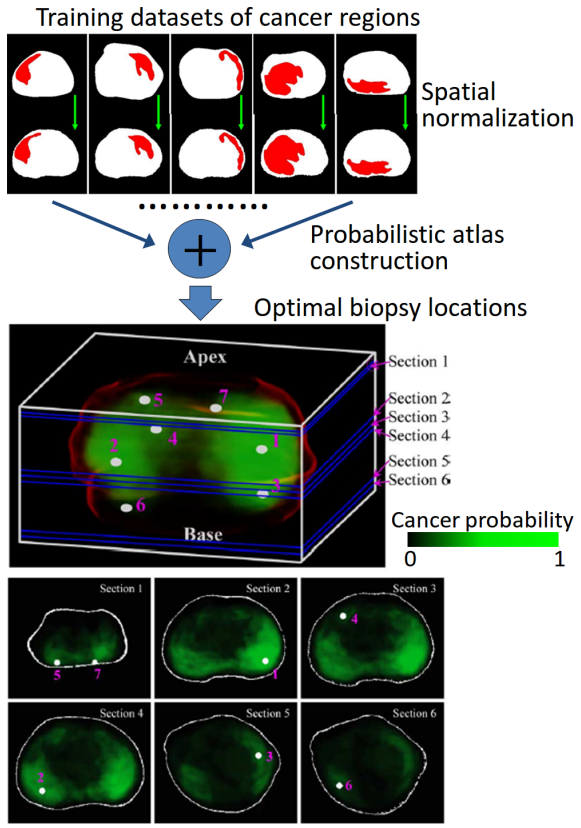

Fig. 4 Optimal prostate biopsy planning based on probabilistic atlas of pathological evidence.

in the standardized prostate space based on the probabilistic atlas [93]. If only one location is used for biopsy, the optimal one has the highest probability in the probabilistic atlas. To select the second location, the probabilistic atlas is constructed for the updated datasets in which the canter tissue does not exist at the first location, and the second optimal location is determined. This process is repeated until the fixed number. The method was extended so as to deal with needle place uncertainty and combined with SVM-based ultrasound image segmentation [94], validated clinically [95], and further extended so as to deal with estimating cancer volume and Gleason Score by combining with SVM [96].

\subsubsection{Optical Biopsy}

Another biopsy application is retargeting in optical biopsy, which enables in vivo visualization of the tissues at microscopic scale without removing tissue samples. In the gastro-intestinal endoscopic optical biopsy, comparison at the same locations in a few month intervals is necessary. For the relocation of biopsy targets, Atasoy et al. applied manifold learning to dimensional reduction in representing endoscopic video sequences for the clustering and classification of the sequences [97], [98]. The developed lowdimensional representations are expected to facilitate targeted optical biopsy.

\subsection{Implant and Reconstructive Surgical Planning}

A large number of surgeries are categorized in a reconstructive surgery, which is regarded a surgery to recover the original anatomy. By anatomy recovery, functional recovery is expected to be attained as a result. Many of implant 
surgeries including artificial joints and stents are performed to artificially reconstruct the original anatomy. Bone fracture reduction is also one of reconstructive surgery. Statistical shape models (SSMs) and other statistical models can be used for prediction of the original anatomy or the best implants realizing it from the pathological anatomy or surrounding health anatomy. In the following, SSM-based approaches to optimizing surgical planning are described for each clinical purpose.

\subsubsection{Total Hip Arthroplasty}

Total hip arthroplasty (THA) is particularly suitable for surgical CAD/CAM paradigm [99]. In the last two decades, the CAM (computer aided manufacturing) part, that is, surgical navigation and robotics, for THA has been well-developed based on registration between intraoperative digitized surface data and preoperative CT surface model. Now that the preoperative plan is accurately executed intraoperatively using the CAM system, the substantial part of THA surgery is now shifted to the CAD part, that is, how preoperative planning is performed. Although interactive systems have been developed for THA planning [100], it still depends on surgeon's expertise. Statistical shape modeling of the relations between the host bone shapes and implant is a promising approach for accurate and objective preoperative planning. Viceconti et al. firstly automated the planning of the femoral stem implant of THA by using one reference planning dataset consisting of 3D CT data and the stem planned on it [101]. Given patient 3D data, scaled rigid registration between reference 3D CT data and the patient 3D data is performed, and then the estimated scale and rigid transformation parameters provide difference from the reference in the stem size and pose. Nakamoto et al. proposed a statistical atlas of the stem implant of THA to automate its planning [102]. This statistical atlas was the average distance map on the implant surface to the medullary cavity of the femur, which is derived from a large number of past plans prepared by an experienced surgeon. Given the patient model of the medullary cavity surface, the size and pose of the stem which minimize the differences in the distance map between the statistical atlas and the actual patient. Figure 5 shows the schematic diagram of automated THA planning based on statistical atlases. Otomaru et al. extended Viceconti's method so as to select the optimal reference plan from a number of planning datasets and compared it with Nakamoto's method using the medullary cavity surface obtained by fully-automated segmentation [103]. Otomaru et al. also automated the acetabular cup planning by using the coupled SSM of the patient pelvis and implanted cup [104] and further combined the merged SSM with the residual bone thickness map similar to the distance map [105] as well as combined with SSM-based 2D-3D reconstruction so as to perform automated THA planning from 2D X-ray images [106]. More recently, Kagiyama et al. automated the all the implant components in THA including the acetabular cup, femoral stem, and neck component

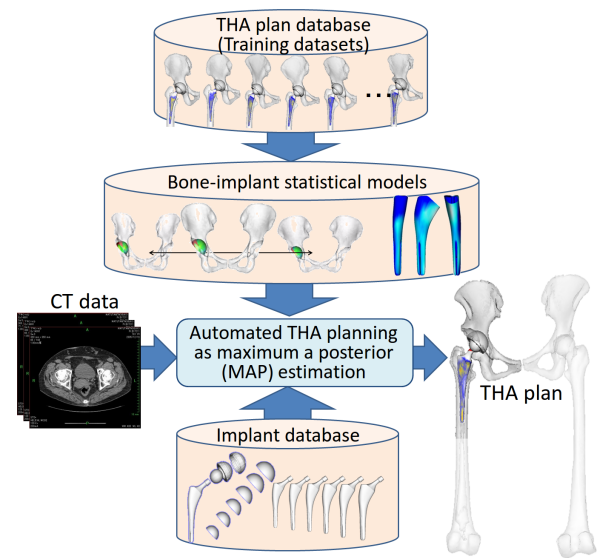

Fig. 5 Automated total hip arthroplasty (THA) planning based on statistical models constructed from THA plan database.

by introducing statistical models of the joint functionality parameters such as the range of motion and limb length difference [107].

In the automated THA planning described above, the statistical models were used to determine the best-fit implant size and position for the specific patient. More recently, Kozic et al. further apply the statistical models to determine the optimal implant shape so as to fit as many patients as possible [108], [109]. The patterns of bone variability among patients are analyzed to improve implant fitting. Results are presented for proximal human tibia [108], [109]. Further, it was shown that the optimized shape can minimize intraoperative deformation of the implant [110].

\subsubsection{Other Applications}

The stent is an implant to recover the original shape of tubular anatomical structures from a deformed one such as stenosis and aneurysm. Pinho et al. predicted the original trachia shape by fitting SSMs to healthy parts of the trachea and used the predicted shape for optimal stenting as well as stenosis quantification [111], [112].

Fracture reduction is another good application of SSMs which are utilized for representing recovered original bone shapes before fracture. The applications to fracture reduction planning of the proximal femur and pelvis have been investigated [113], [114], where the problem was formulated as finding the best pose parameters of the bone fragments and shape parameters of the SSM, representing the original bone shape, which best fit to the surfaces of the bone fragments. Gong et al. combined the above approach with 2D-3D reconstruction to realize 2D X-ray image based fracture reduction planning of the wrist joint bones whose shapes are simpler than the hip joint bones [115].

SSMs are applied to patient-specific catheterization planning. The shape of the guidewire, which precedes the catheter, is crucial to ensure smooth insertion to the target branch. In order to effectively reduce the search space, the guidewire shape is modeled as SSM which was constructed 
from the training datasets of guidewire shapes generated by experienced interventional radiologists [116]. Furthermore, physics simulations of catheterization were combined to obtain the optimal guidewire shape [117].

\subsection{Radiation Therapy}

Statistical modeling in radiation therapy planning has been mainly applied to segmentation and motion estimation of the target and organs at risk (OARs) from 3D data [118][120]. In addition, Prince et al. utilized SSMs for obtaining coverage probability map representing the uncertainties during the radiotherapy process [121]. More recently, intensity modulated radiation therapy (IMRT) has become available, by which more precise radiation planning is possible. By the optimized radiation using IMRT, it is possible to deliver a higher dose of radiation to the tumor while sparing nearby OARs. However, optimized planning which realizes such an ideal radiation also becomes more complex. Simari et al. proposed to use the radiation therapy plan database of past patients for efficient optimization of the plan of a new patient using a statistical approach [122]. A shape relation descriptor, the overlap volume histogram $(\mathrm{OVH})$, was proposed [123] and the statistical features of OVH were used to query for the lowest achievable dose [122].

\section{Conclusion}

We have described a survey of applications of statistical modeling and machine learning to computer assisted medical intervention. The reviewed articles are mainly related to intraoperative recovery of shape, motion, and deformation of anatomical structures from incomplete and sparse data as obtained during intervention. Statistical modeling and machine learning have been effectively used for modeling prior knowledge required for their recovery. Biomechanical simulations are also combined with intraoperative data to recover biomechanically-justified deformations even when boundary conditions are incomplete through statistical modeling and machine learning.

One obvious important goal of computer assisted intervention is to optimize the intervention. Statistical modeling and machine learning have also been effective for the optimization. As a future perspective, this direction will greatly expand due to accumulation of large amount of highresolution and accurate datasets during preoperative imaging and examination, intraoperative operation and sensing, and postoperative follow-up. These datasets will be used for training datasets of machine learning to optimize the intervention. The articles reviewed in this paper utilized only a part of these datasets, and future researches will try to fully utilize them to optimize the intervention so as to provide the best outcome.

\section{Acknowledgments}

The authors of this paper are partly supported by MEXT
Grant-in-Aid for Scientific Research on Innovative Areas "Computational Anatomy for Computer-aided Diagnosis and Therapy: Frontiers of Medical Image Sciences".

References

[1] R.H. Taylor and P. Kazanzides, "Medical robotics and computerintegrated interventional medicine," Advances in Computers, vol.73, pp.219-260, 2008.

[2] A. Liu, F. Tendick, K. Cleary, and C. Kaufmann, "A survey of surgical simulation: Applications, technology, and education," Presence, vol.12, no.6, pp.599-614, 2003.

[3] H. Delingette, X. Pennec, L. Soler, J. Marescaux, and N. Ayache, "Computational models for image-guided robot-assisted and simulated medical interventions," Proc. IEEE, vol.94, no.9, pp.16781688, 2006.

[4] T. Heimann and H-P. Meinzer "Statistical shape models for 3D medical image segmentation: A review," Medical Image Analysis, vol.13, no.4, pp.543-563, 2009.

[5] H. Müller, N. Michoux, D. Bandon, and A. Geissbuhler, "A review of content-based image retrieval systems in medical applicationsclinical benefits and future directions," Int. J. Medical Informatics, vol.73, no.1, pp.1-23, 2004.

[6] D.J. Hawkes, D. Barratt, J.M. Blackall, C. Chan, P.J. Edwards, K. Rhode, G.P. Penney, J. McClelland, and D.L.G. Hill, "Tissue deformation and shape models in image-guided interventions: A discussion paper," Medical Image Analysis, vol.9, no.2, pp.163-175, 2005.

[7] J. Rosen, B. Hannaford, C.G. Richards, and M.N. Sinanan, "Markov modeling of minimally invasive surgery based on tool/tissue interaction and force/torque signatures for evaluating surgical skills," IEEE Trans. Biomed. Eng., vol.48, no.5, pp.579-591, 2001.

[8] G. Megali, S. Sinigaglia, O. Tonet, and P. Dario, "Modelling and evaluation of surgical performance using hidden Markov models," IEEE Trans. Biomed. Eng., vol.53, no.10, pp.1911-1999, 2001.

[9] N. Padoy, T. Blum, S.A. Ahmadi, H. Feussner, M.O. Berger, and N. Navab, "Statistical modeling and recognition of surgical workflow," Medical Image Analysis, vol.16, no.3, pp.632-641, 2012.

[10] J.A. Noble and D. Boukerroui, "Ultrasound image segmentation: A survey," IEEE Trans. Med. Imaging, vol.25, no.8, pp.987-1010, 2006.

[11] M. Fleute and S. Lavallé, "Building a complete surface model from sparse data using statistical shape models: Application to computer assisted knee surgery," Proc. 1st International Conference on Medical Image Computing and Computer Assisted Intervention (MICCAI 1998), Boston, MA, Lect. Notes Comput. Sci., vol.1496, pp.879-887, 1998.

[12] M. Fleute, S. Lavallé, and R. Julliard, "Incorporating a statistically based shape model into a system for computer-assisted anterior cruciate ligament surgery," Medical Image Analysis, vol.3, no.3, pp.209-222, 1999.

[13] T.F. Cootes, C.J. Taylor, D.H. Cooper, and J. Graham, "Active shape models-their training and application," Comput. Vis. Image Understand., vol.61, no.1, pp.38-59, 1995.

[14] E. Stindel, J.L. Briard, P. Merloz, S. Plaweski, F. Dubrana, C. Lefevre, and J. Troccaz, "Bone morphing: 3D morphological data for total knee arthroplasty," Computer Aided Surgery, vol.7, pp.156-168, 2002.

[15] P. Markelj, D. Tomažvič, B. Likar, and F. Pernuš, "A review of 3D/2D registration methods for image-guided interventions," Medical Image Analysis, vol.16, no.3, pp.642-661, 2012.

[16] M. Fleute and S. Lavallé, "Nonrigid 3-d/2-d registration of images using statistical models," Proc. 2nd International Conference on Medical Image Computing and Computer Assisted Intervention (MICCAI 1999), Cambridge, UK, Lect. Notes Comput. Sci., 
vol.1679, pp.138-147, 1999.

[17] M. Fleute, S. Lavallé, and L. Desbat, "Integrated approach for matching statistical shape models with intra-operative 2D and 3D data," Proc. 5th International Conference on Medical Image Computing and Computer Assisted Intervention (MICCAI 2002), Tokyo, Japan, Lect. Notes Comput. Sci., vol.2489, pp.364-372, 2002.

[18] S. Benameur, M. Mignotte, S. Parent, H. Labelle, W. Skalli, and J.A. De Guise, "3D biplanar reconstruction of scoliotic vertebrae using statistical models," Proc. IEEE Computer Society Conference on Computer Vision and Pattern Recognition (CVPR 2001), vol.2, pp.577-582, 2001.

[19] S. Benameur, M. Mignotte, S. Parent, H. Labelle, W. Skalli, and J.A. De Guise, "3D/2D registration and segmentation of scoliotic vertebrae using statistical models," Computerized Medical Imaging and Graphics, vol.25, no.5, pp.321-337, 2003.

[20] S. Benameur, M. Mignotte, H. Labelle, and J.A. De Guise, "A hierarchical statistical modeling approach for the unsupervised 3-D biplanar reconstruction of the scoliotic spine," IEEE Trans. Biomed. Eng., vol.52, no.12, pp.2041-2057, 2005.

[21] J. Boisvert, F. Cheriet, X. Pennec, H. Labelle, and N. Ayache, "Geometric variability of the scoliotic spine using statistics on articulated shape models," IEEE Trans. Med. Imaging, vol.27, no.4, pp.557-568, 2008.

[22] H. Lamecker, T.H. Wenckebach, and H-C. Hege, "Atlas-based 3Dshape reconstruction from X-ray images," Proc 18th International Conference on Pattern Recognition (ICPR 2006), pp.371-374, 2006.

[23] G. Zheng, M.Á.G. Ballester, M. Styner, and L-P. Nolte, "Reconstruction of patient-specific 3D bone surface from 2D calibrated fluoroscopic images and point distribution model," Proc. 9th International Conference on Medical Image Computing and Computer Assisted Intervention (MICCAI 2006), Copenhagen, Denmark, Lect. Notes Comput. Sci., vol.4190, pp.25-32, 2006.

[24] G. Zheng, S. Gollmer, S. Schumann, X. Dong, T. Feilkas, and M.Á.G. Ballester, "A 2D/3D correspondence building method for reconstruction of a patient-specific 3D bone surface model using point distribution models and calibrated X-ray images," Medical Image Analysis, vol.13, no.6, pp.883-899, 2009.

[25] R. Kurazume, K. Nakamura, T. Okada, Y. Sato, N. Sugano, T. Koyama, Y. Iwashita, and T. Hasegawa, "3D reconstruction of a femoral shape using a parametric model and two 2D fluoroscopic images," Comput. Vis. Image Understand., vol.113, no.2, pp.202-211, 2009.

[26] T. Evgeniou, M. Pontil, and T. Poggio, "Regularization networks and support vector machines," Advances in Computational Mathematics, vol.13, no.1, pp.1-50, 2000.

[27] G. Zheng, "Statistically deformable 2D/3D registration for accurate determination of post-operative cup orientation from single standard X-ray radiograph,” Proc. 12th International Conference on Medical Image Computing and Computer Assisted Intervention (MICCAI 2009), Part I, London, UK, Lect. Notes Comput. Sci., vol.5761, pp.820-827, 2009.

[28] G. Zheng, "Statistically deformable 2D/3D registration for estimating post-operative cup orientation from a single standard AP X-ray radiograph," Annals of Biomedical Engineering, vol.38, no.9, pp.2910-2927, 2010.

[29] G. Zheng, L-P. Nolte, and S.J. Ferguson, "Scaled, patient-specific 3D vertebral model reconstruction based on 2D lateral fluoroscopy," Int. J. Computer Assisted Radiology and Surgery, vol.6, no.3, pp.351-366, 2011.

[30] J. Yao and R.H. Taylor, "Tetrahedral mesh modeling of density data for anatomical atlases and intensity-based registration," Proc. 3rd International Conference on Medical Image Computing and Computer Assisted Intervention (MICCAI 2000), Pittsburg, PA, Lect. Notes Comput. Sci., vol.1935, pp.663-669, 2000.

[31] J. Yao and R.H. Taylor, "Assessing accuracy factors in deformable
2D/3D medical image registration using a statistical pelvis model," Proc. IEEE International Conference on Computer Vision (ICCV 2003), vol.2, pp.1329-1334, 2003.

[32] G. Chintalapani, L.M. Ellingsen, O. Sadowsky, J.L. Prince, and R.H. Taylor, "Statistical atlases of bone anatomy: construction, iterative improvement and validation," Proc. 10th International Conference on Medical Image Computing and Computer Assisted Intervention (MICCAI 2007), Brisbane, Australia, Lect. Notes Comput. Sci., vol.4791, pp.499-506, 2007.

[33] O. Sadowsky, G. Chintalapani, and R.H. Taylor "Deformable 2D-3D registration of the pelvis with a limited field of view, using shape statistics," Proc. 10th International Conference on Medical Image Computing and Computer Assisted Intervention (MICCAI 2007), Brisbane, Australia, Lect. Notes Comput. Sci., vol.4792, pp.519-526, 2007.

[34] O. Sadowsky, L. Junghoon, F.G. Sutter, S.J. Wall, J.L. Prince, and R.H. Taylor "Hybrid cone-beam tomographic reconstruction: Incorporation of prior anatomical models to compensate for missing data," IEEE Trans. Med. Imaging, vol.30, no.1, pp.69-83, 2011.

[35] C.S.K. Chan, D.C. Barratt, P.J. Edwards, G.P. Penney, M. Slomczykowski, T.J. Carter, and D.J. Hawkes, "Cadaver validation of the use of ultrasound for 3D model instantiation of bony anatomy in image guided orthopaedic surgery," Proc. 7th International Conference on Medical Image Computing and Computer Assisted Intervention (MICCAI 2007), St Malo, France, Lect. Notes Comput. Sci., vol.3217, pp.397-404, 2004.

[36] D.C. Barratt, C.S.K. Chan, P.J. Edwards, G.P. Penney, M. Slomczykowski, T.J. Carter, and D.J. Hawkes, "Instantiation and registration of statistical shape models of the femur and pelvis using 3D ultrasound imaging," Medical Image Analysis, vol.12, pp.358-374, 2008.

[37] H. Talib, K. Rajamani, J. Kowal, L-P. Nolte, M. Styner, and M.A. Gonzalez Ballester, "A comparison study assessing the feasibility of ultrasound-initialized deformable bone models," Computer Aided Surgery, vol.10, no.5/6, pp.293-299, 2005.

[38] K.T. Rajamani, M.A. Styner, H. Talib, G. Zheng, L-P. Nolte, and M.A. Gonzalez Ballester, "Statistical deformable bone models for robust 3D surface extrapolation from sparse data," Medical Image Analysis, vol.11, pp.99-109, 2007.

[39] G. Zheng, X. Dong, K.T. Rajamani, X. Zhang, M. Styner, R.U. Thoranaghatte, L-P. Nolte, and M.A. Gonzalez Ballester, "Accurate and robust reconstruction of a surface model of the proximal femur from sparse-point data and a dense-point distribution model for surgical navigation,” IEEE Trans. Biomed. Eng., vol.54, no.12, pp.2109-2122, 2007.

[40] S. Khallaghi, P. Mousavi, R.H. Gong, S. Gill, J. Boisvert, G. Fichtinger, D. Pichora, D. Borschneck, and P. Abomaesumi, "Registration of a statistical shape model of the lumbar spine to 3D ultrasound images," Proc. 13th International Conference on Medical Image Computing and Computer Assisted Intervention (MICCAI2010), Part II, Beijing, China, Lect. Notes Comput. Sci., vol.6362, pp.68-75, 2010.

[41] P. Foroughi, D. Song, G. Chintalapani, R.H. Taylor, and G. Fichtinger, "Localization of pelvic anatomical coordinate system using US/atlas registration for total hip replacement," Proc. 11th International Conference on Medical Image Computing and Computer Assisted Intervention (MICCAI2008), Part II, New York City, Lect. Notes Comput. Sci., vol.5542, pp.871-879, 2008.

[42] S. Scumann, M. Puls, T. Ecker, T. Schwaegli, J. Stifter, K-A. Siebenrock, and G. Zheng, "Determination of pelvic orientation from ultrasound images using patch-SSMs and a hierarchical speed of sound compensation strategy," Proc. Information Processing in Computer Assisted Intervention (IPCAI 2010), Lect. Notes Comput. Sci., vol.6135, pp.157-167, 2010.

[43] S. Scumann, L-P. Nolte, and G. Zheng, "Compensation of sound speed deviations in 3D B-mode ultrasound for intraoperative determination of the anterior pelvic plane," IEEE Trans. Information 
Technology and Biomedicine, vol.16, no.1, pp.88-97, 2012.

[44] I. Hacihaliloglu, R. Abugharbieh, A.J. Hodgson, and R.N. Rohling, "Bone surface localization in ultrasound using image phase-based features," Ultrasound in Medicine and Biology, vol.35, no.9, pp.1475-1487, 2009.

[45] S. Ghanavati, P. Mousavi, G. Fichtinger, P. Foroughi, and P. Abolmaesumi, "Multi-slice to volume registration of ultrasound data to a statistical atlas of human pelvis," Proc. SPIE Medical Imaging 2010: Visualization, Image-Guided Procedure, and Modeling, vol.7625, 76250O-1, 2010.

[46] C. Davatzikos, D. Shen, A. Mohamed, and S.K. Kyriacou, "A framework for predictive modeling of anatomical deformations," IEEE Trans. Med. Imaging, vol.20, no.8, pp.836-843, 2001.

[47] S. Liao, H. Jia, G. Wu, and D. Shen, "A novel framework for longitudinal atlas construction with groupwise registration of subject image sequences," NeuroImage, vol.59, no.2, pp.1275-1289, 2012.

[48] A. Serag, P. Aljabar, G. Ball, S.J. Counsell, J.P. Boardman, M.A. Rutherford, A.D. Edwards, J.V. Hajnal, and D. Rueckert, "Construction of a consistent high-definition spatio-temporal atlas of the developing brain using adaptive kernel regression," NeuroImage, vol.59, no.3, pp.2255-2265, 2012.

[49] M. Prastawa, S.P. Awate, and G. Gerig, "Building spatiotemporal anatomical models using joint 4-D segmentation, registration, and subject-specific atlas estimation," Proc. 2012 IEEE Workshop on Mathematical Methods in Biomedical Image Analysis, pp.49-56, 2012.

[50] D. Perperidis, R. Mohiaddin, and D. Rueckert, "Construction of a 4D statistical atlas of the cardiac anatomy and its use in classification," Proc. 8th International Conference on Medical Image Computing and Computer Assisted Intervention (MICCAI 2005), pp.402-410, 2005.

[51] N. Duchateau, M.D. Craene, G. Piella, E. Silva, A. Doltra, M. Sitges, B.H. Bijnens, and A.F. Frangi, "A spatiotemporal statistical atlas of motion for the quantification of abnormal myocardial tissue velocities," Medical Image Analysis, vol.15, no.3, pp.316-328, 2011.

[52] Q. Zhang, A. Pevsner, A. Hertanto, Y.C. Hu, K.E. Rosenzweig, C.C. Ling, and G.S. Mageras, "A patient-specific respiratory model of anatomical motion for radiation treatment planning," Medical Physics, vol.34 no.12, pp.4772-4781, 2007.

[53] Q. Zhang, Y.C. Hu, F. Liu, K. Goodman, K.E. Rosenzweig, and G.S. Mageras, "Correction of motion artifacts in cone-beam CT using a patient-specific respiratory motion model," Medical Physics, vol.37 no.6, pp.2901-2909, 2010.

[54] X. Liu, I. Oguz, S.M. Pizer, and G.S. Mageras, "Shape-correlated deformation statistics for respiratory motion prediction in 4D lung," Proc. SPIE Medical Imaging 2010: Visualization, ImageGuided Procedures, and Modeling, vol.7625, pp.76252D-76252D$10,2010$.

[55] J. Ehrhardt, R. Werner, A. Schmidt-Richberg, B. Schulz, and H. Handels, "Generation of a mean motion model of the lung using 4D-CT image data," Proc. Eurographics Workshop on Visual Computing for Biomedicine, pp.69-76, 2008.

[56] J. Ehrhardt, R. Werner, A. Schmidt-Richberg, and H. Handels, "Statistical modeling of 4D respiratory lung motion using diffeomorphic image registration," IEEE Trans. Med. Imaging, vol.30, no.2, pp.251-265, 2011.

[57] R. Barnett, S. Meikle, and R. Fulton, "Statistical motion modeling of the thorax applied to respiratory gated FDG PET," Proc. IEEE Nuclear Science Symposium \& Medical Imaging Conference, pp.2459-2464. 2010

[58] M. Nakamoto, Y. Sato, H. Shiono, and Y. Sawa, "Registration of the trachea using skin surface matching and statistical bias correction for computer assisted mediastinoscopy," Int. J. Computer Assisted Radiology and Surgery, vol.3, no.S1, pp.S103-S118, 2008

[59] A.P. King, J.M. Blackall, G.P. Penney, and D.J. Hawkes, "Tracking liver motion using 3-D ultrasound and a surface based statistical shape model," Proc. IEEE Workshop on Mathematical Methods in Biomedical Image Analysis (MMBIA 2001), pp.145-152, 2001.

[60] J.M. Blackall, A.P. King, G.P. Penney, A. Adam, and D.J. Hawkes, "A statistical model of respiratory motion and deformation of the liver," Proc. 4th International Conference on Medical Image Computing and Computer Assisted Intervention (MICCAI 2001), vol.2208, pp.1338-1340, 2001.

[61] M. von Siebenthal, G. Székely, A. Lomax, and P.C. Cattin, "Intersubject modelling of liver deformation during radiation therapy," Proc. 10th International Conference on Medical Image Computing and Computer Assisted Intervention (MICCAI 2007), vol.4791, pp.659-666, 2007.

[62] P. Arnold, F. Preiswerk, B. Fasel, R. Salomir, K. Scheffler, and P.C. Cattin, "3D organ motion prediction for MR-guided high intensity focused ultrasound," Proc. 14th International Conference on Medical Image Computing and Computer Assisted Intervention (MICCAI 2011), vol.6892, pp.623-630, 2011.

[63] S.-L. Lee, A. Chung, M. Lerotic, M.A. Hawkins, D. Tait, and G.-Z. Yang, "Dynamic shape instantiation for intra-operative guidance," Proc. 13th International Conference on Medical Image Computing and Computer Assisted Intervention (MICCAI 2010), vol.6361, pp.69-76, 2010

[64] S.L. Lee, C. Riga, L. Crowie, M. Hamady, N. Cheshire, and G.Z. Yang, "An instantiability index for intra-operative tracking of 3D anatomy and interventional devices," Proc. Medical Image Computing and Computer-Assisted Intervention - MICCAI 2011, vol.6891, pp.49-56, 2011.

[65] C. Basdogan, M. Sedef, M. Harders, and S. Wesarg, "VR-based simulators for training in minimally invasive surgery," IEEE Comput. Graph. Appl., vol.27, no.2, pp.54-66, 2007.

[66] U. Meier, O. López, C. Monserrat, M.C. Juan, and M. Alcañiz, "Real-time deformable models for surgery simulation: A survey," Computer Methods and Programs in Biomedicine, vol.77, no.3, pp.183-197, 2005.

[67] R. Kikuuwe, H. Tabuchi, and M. Yamamoto, "An edge-based computationally efficient formulation of saint Venant-Kirchhoff tetrahedral finite elements," ACM Trans. Graph., vol.28, no.1, pp.8:1-8:13, 2009.

[68] M. Müller, J. Dorsey, L. McMillan, R. Jagnow, and B. Cutler, "Stable real-time deformations," Proc. 2002 ACM SIGGRAPH/ Eurographics Symposium on Computer Animation, pp.49-54, 2002.

[69] L.B. Querol, P. Büchler, D. Rueckert, L.P. Nolte, and M.Á.G. Ballester, "Statistical finite element model for bone shape and biomechanical properties," Proc. Medical Image Computing and Computer-Assisted Intervention - MICCAI 2006, vol.4190, pp.405-411, 2006.

[70] R. Huiskes and E.Y. Chao, "A survey of finite element analysis in orthopedic biomechanics: The first decade," J. Biomechanics, vol.16, no.6, pp.385-409, 1983.

[71] S. Gill, P. Abolmaesumi, G. Fichtinger, J. Boisvert, D. Pichora, D. Borshneck, and P. Mousavi, "Biomechanically constrained groupwise ultrasound to CT registration of the lumbar spine," Medical Image Analysis, vol.16, no.3, pp.662-674, 2012.

[72] C.K. Fitzpatrick, M.A. Baldwin, P.J. Rullkoetter, and P.J. Laz, "Combined probabilistic and principal component analysis approach for multivariate sensitivity evaluation and application to implanted patellofemoral mechanics," J. Biomechanics vol.44, no.1, pp.13-21, 2011.

[73] P. Dumpuri, R.C. Thompson, T.K. Sinha, and M.I. Miga, "Automated brain shift correction using a pre-computed deformation atlas," Proc. SPIE Medical Imaging 2006: Visualization, ImageGuided Procedures, and Display, vol.6141, pp.430-437, 2006.

[74] K. Paulsen, M. Miga, F. Kennedy, P. Hoopes, A. Hartov, and D. Roberts, "A computational model for tracking subsurface tissue deformation during stereotactic neurosurgery," IEEE Trans. Biomed. 
Eng., vol.46, no.2, pp.213-225, 1999.

[75] A. Mohamed and C. Davatzikos, "Finite element modeling of brain tumor mass-effect from 3D medical images," Proc. Medical Image Computing and Computer-Assisted Intervention - MICCAI 2005, vol.3749, pp.400-408, 2005.

[76] A. Mohamed, E.I. Zacharaki, D. Shen, and C. Davatzikos, "Deformable registration of brain tumor images via a statistical model of tumor-induced deformation," Medical Image Analysis, vol.10, no.5, pp.752-763, 2006.

[77] A. Mohamed, C. Davatzikos, and R.H. Taylor, "A combined statistical and biomechanical model for estimation of intraoperative prostate deformation," Proc. Medical Image Computing and Computer-Assisted Intervention - MICCAI 2002, vol.2489, pp.452-460, 2002.

[78] Y. Hu, R. van den Boom, T. Carter, Z. Taylor, D. Hawkes, H.U. Ahmed, M. Emberton, C. Allen, and D. Barratt, "A comparison of the accuracy of statistical models of prostate motion trained using data from biomechanical simulations," Progress in Biophysics \& Molecular Biology, vol.103, no.2-3, pp.262-272, 2010.

[79] Y. Hu, T. Carter, H.U. Ahmed, M. Emberton, C. Allen, D. Hawkes, and D. Barratt, "Modelling prostate motion for data fusion during image-guided interventions," IEEE Trans. Med. Imaging, vol.30, no.11, pp.1887-1900, 2011

[80] Y. Hu, H.U. Ahmed, Z. Taylor, C. Allen, M. Emberton, D. Hawkes, and D. Barratt, "MR to ultrasound registration for image-guided prostate interventions," Medical Image Analysis, vol.16, no.3, pp.687-703, 2012

[81] C. Tanner, J.H. Hipwell, and D.J. Hawkes, "Using statistical deformation models for the registration of multimodal breast images," Proc. SPIE Medical Imaging 2009: Image Processing, vol.7259, pp.72590P-72590P-9, 2009.

[82] Q. He, H.H.S. Ip, J. Feng, and X. Cao, "Mr-SDM: A novel statistical deformable model for object deformation," The Visual Computer, vol.25, no.5-7, pp.609-616, 2009.

[83] Q. He, J. Feng, H.H.S. Ip, J. Xia, and X. Cao, "An integration of statistical deformable model and finite element method for bonerelated soft tissue prediction in orthognathic surgery planning," Proc. 4th International Workshop on Medical Imaging and Augmented Reality, pp.31-39, 2008.

[84] R. Sierra, J. Zátonyi, M. Bajka, G. Székely, and M. Harders, "Hydrometra simulation for VR-based hysteroscopy training," Proc. Medical Image Computing and Computer-Assisted Intervention MICCAI 2005, vol.3750, pp.575-582, 2005.

[85] M. Harders and G. Székely, "Using statistical shape analysis for the determination of uterine deformation states during hydrometra," Proc. Medical Image Computing and Computer-Assisted Intervention - MICCAI 2007, vol.4792, pp.858-865, 2007.

[86] S.R. Mousavi, I. Khalaji, A.S. Naini, K. Raahemifar, and A. Samani, "Statistical finite element method for real-time tissue mechanics analysis," Computer Methods in Biomechanics and Biomedical Engineering, vol.15, no.6, pp.595-608, 2012.

[87] I. Khalaji, K. Rahemifar, and A. Samani, "Accelerated statistical shape model-based technique for tissue deformation estimation," Proc. SPIE Medical Imaging 2009: Visualization, Image-Guided Procedures, and Display, vol.7261, pp.72610U-72610U-9, 2009.

[88] K. Morooka, X. Chen, R. Kurazume, S. Uchida, K. Hara, Y. Iwashita, and M. Hashizume, "Real-time nonlinear FEM with neural network for simulating soft organ model deformation," Proc. Medical Image Computing and Computer-Assisted Intervention MICCAI 2008, vol.5242, pp.742-749, 2008.

[89] K. Morooka, T. Taguchi, X. Chen, R. Kurazume, M. Hashizume, and T. Hasegawa, "A method for constructing real-time FEMbased simulator of stomach behavior with large-scale deformation by neural networks," Proc. SPIE Medical Imaging 2012: Image-Guided Procedures, Robotic Interventions, and Modeling, vol.8316, pp.83160J-83160J-6, 2012.

[90] G. Bianchi, B. Solenthaler, G. Székely, and M. Harders,
"Simultaneous topology and stiffness identification for massspring models," Proc. Medical Image Computing and ComputerAssisted Intervention - MICCAI 2004, vol.3217, pp.293-301, 2004.

[91] G. Sela, J. Subag, A. Lindblad, D. Albocher, S. Schein, and G. Elber, "Real-time haptic incision simulation using FEM-based discontinuous free-form deformation," Comput.-Aided Des., vol.39, no.8, pp.685-693, 2007.

[92] D. Shen, Z. Lao, J. Zeng, E.H. Herskovits, G. Fichtinger, and C. Davatzikos, "A statistical atlas of prostate cancer for optimal biopsy," Proc. 4th International Conference on Medical Image Computing and Computer Assisted Intervention (MICCAI 2001), Utrecht, Netherlands, Lect. Notes Comput. Sci., vol.2208, pp.416-424, 2001.

[93] D. Shen, Z. Lao, J. Zeng, W. Zhang, I.A. Sesterhenn, L. Sun, J.W. Moul, E.H. Herskovits, G. Fichtinger, and C. Davatzikos, "Optimized prostate biopsy via a statistical atlas of cancer spatial distribution," Medical Image Analysis, vol.8, pp.139-150, 2004.

[94] Y. Zhan, D. Shen, J. Zeng, W. Zhang, I.A. Sesterhenn, L. Sun, J.W. Moul, E.H. Herskovits, G. Fichtinger, and C. Davatzikos, "Targeted prostate biopsy using statistical image analysis," IEEE Trans. Med. Imaging, vol.26, no.6, pp.779-788, 2007.

[95] R. Narayanan, P.N. Werahera, A. Barqawi, E.D. Crawford, K. Shinohara, A.R. Simoneau, and J.S. Suri, "Adaptation of a 3D prostate cancer atlas for transrectal ultrasound guided targetspecific biopsy," Physics in Biology and Medicine, vol.53, no.20, pp.N397-406, 2008.

[96] Y. Ou, D. Shen, J. Zeng, L. Sun, J.W. Moul, and C. Davatzikos, "Sampling the spatial patterns of cancer: Optimized biopsy procedures for estimating prostate cancer volume and Gleason Score," Medical Image Analysis, vol.13, pp.609-620, 2009.

[97] S. Atasoy, B. Glocker, S. Giannarou, D. Mateus, A. Meining, G-Z. Yang, and N. Navab, "Probabilistic region matching in narrowband endoscopy for targeted optical biopsy," Proc. 12th International Conference on Medical Image Computing and Computer Assisted Intervention (MICCAI2008), London, UK, Lect. Notes Comput. Sci., vol.5761, pp.499-506, 2009.

[98] S. Atasoy, D. Mateus, A. Meining, G-Z. Yang, and N. Navab, "Endoscopic video manifolds for targeted optical biopsy," IEEE Trans. Med. Imaging, vol.31, no.3, pp.637-653, 2012.

[99] R.H. Taylor and D. Stoianovici, "Medical robotics in computerintegrated surgery," IEEE Trans. Robot. Autom., vol.19, no.5, pp.765-781, 1994.

[100] M. Viceconti, R. Lattanzi, B. Antonietti, S. Paderni, R. Olmi, A. Sudanese, and A. Toni, "CT-based surgical planning software improves the accuracy of total hip replacement preoperative planning," Medical Engineering and Physics, vol.25, no.5, pp.371-377, 2003.

[101] M. Viceconti, D. Testi, M. Simeoni, and C. Zannoni, "An automated method to position prosthetic components within multiple anatomical spaces," Computer Methods and Programs in Biomedicine, vol.70, pp.121-127, 2003.

[102] M. Nakamoto, I. Otomaru, M. Takao, N. Sugano, Y. Kagiyama, H. Yoshikawa, Y. Tada, and Y. Sato, "Construction of a statistical surgical plan atlas for automated 3D planning of femoral component in total hip arthroplasty," Proc. 11th International Conference on Medical Image Computing and Computer Assisted Intervention (MICCAI2008), Part II, New York City, Lect. Notes Comput. Sci., vol.5542, 718-725, 2008.

[103] I. Otomaru, M. Nakamoto, Y. Kagiyama, M. Takao, N. Sugano, N. Tomiyama, Y. Tada, and Y. Sato, "Automated preoperative planning of femoral stem in total hip arthroplasty from 3D CT data: Atlas-based approach and comparative study," Medical Image Analysis, vol.16, no.2, pp.415-426, 2012.

[104] I. Otomaru, K. Kobayashi, T. Okada, M. Nakamoto, M. Takao, N. Sugano, Y. Tada, and Y. Sato, "CT-based automated preoperative planning of acetabular cup size and position using pelvis-cup 
integrated statistical shape model," Proc. Annual Meeting of International Society on Computer Assisted Orthopaedic Surgery (CAOS 2009), pp.185-188, Boston, MA, 2009.

[105] I. Otomaru, K. Kobayashi, T. Okada, M. Nakamoto, Y. Kagiyama, M. Takao, N. Sugano, Y. Tada, and Y. Sato, "Expertise modeling for automated planning of acetabular cup in total hip arthroplasty using combined bone and implant statistical atlases," Proc. 12th International Conference on Medical Image Computing and Computer Assisted Intervention (MICCAI 2009), Part I, London, UK, Lect. Notes Comput. Sci., vol.5761, pp.532-539, 2009.

[106] I. Otomaru, G. Zheng, M. Takao, N. Sugano, Y. Tada, N. Tomiyama, L-P. Nolte, N. Sugano, and Y. Sato, "An automated 3D cup planning in total hip arthroplasty from a standard $\mathrm{X}$ ray radiograph using atlas-based 2D-3D pelvis shape reconstruction," Proc. Annual Meeting of International Society on Computer Assisted Orthopaedic Surgery (CAOS 2011), London, UK, 17, 2011

[107] Y. Kagiyama, I. Otomaru, M. Takao, N. Sugano, Y. Minakuchi, Y. Tada, N. Tomiyama, and Y. Sato, "Automated CT-based THA planning for optimizing joint functionalities: A maximum a posterior (MAP) estimation approach," Proc. Annual Meeting of International Society on Computer Assisted Orthopaedic Surgery, Boston, MA, (CAOS 2012), 2012.

[108] N. Kozic, M.Á. González Ballester, M. Tannast, L-P. Nolte, and M. Reyes, "Statistical shape space analysis based on level sets," Proc. Medical Imaging and Augmented Reality (MIAR 2008), Lect. Notes Comput. Sci., vol.5128, pp.160-167, 2008.

[109] N. Kozic, S. Weber, P. Buchler, L-P. Nolte, N. Reimers, M.A.G. Ballester, and M. Reyes, "Optimisation of orthopaedic implant design using statistical shape space analysis based on level sets," Medical Image Analysis, vol.14, pp.265-275, 2010.

[110] H. Bou-Sleiman, L.E. Ritacco, L-P. Nolte, and M. Reyes "Minimization of intra-operative shaping of orthopaedic fixation plates: a population-based design," Proc. 14th International Conference on Medical Image Computing and Computer Assisted Intervention (MICCAI 2011), Toronto, Canada, Lect. Notes Comput. Sci., vol.6892, pp.409-416, 2011.

[111] R. Pinho, T. Huysmans, W. Vos, and J. Sijbers, "Tracheal stent prediction using statistical deformable models of tubular shapes," Proc. SPIE Medical Imaging 2008: Image Processing, vol.6914, 691440-1, 2008.

[112] R. Pinho, K.G. Tournoy, and J. Sijbers, "Assessment and stenting of tracheal stenosis using deformable shape models," Medical Image Analysis, vol.15, pp.250-266, 2011.

[113] M.H. Moghari and P. Abolmaesumi, "Global registration of multiple bone fragments using statistical atlas models: feasibility experiments," Proc. 30th Annual International IEEE EMBS Conference, pp.5373-5377, Vancouver, BC, Canada, 2008.

[114] A.W. Turner, A.I. Nahkla, B.L. Davies, Y. Rodriguez, and F.M. Baena, "Acetabular fracture reduction planning using statistical shape models," Proc. Annual Meeting of International Society on Computer Assisted Orthopaedic Surgery (CAOS 2009), pp.169-172, Boston, MA, 2009.

[115] R.H. Gong, J. Stewart, and P. Abolmaesumi, "Multiple-object 2D-3D registration for non-invasive pose identification of fracture fragments," IEEE Trans. Biomed. Eng., vol.58, no.6, pp.1592$1601,2011$.

[116] T. Asaka, T. Okada, K. Osuga, M. Hori, Y-W. Chen, H. Nakamura, and Y. Sato, "Determination of optimal guidewire shape for catheterization procedure based on vessel shape at bifurcation," Int. J. Computer Assisted Radiology, vol.3, Suppl.1, pp.S66-S67, 2008.

[117] S. Hirayama, T. Okada, K. Osuga, M. Hori, Y-W. Chen, N. Tomiyama, and Y. Sato, "Simulation-based patient-specific prediction of good guidewire shape in catheterization," Int. J. Computer Assisted Radiology, vol.7, Suppl.1, pp.S7-S11, 2012.

[118] V. Pekar, T.R. McNutt, and M.R. Kaus, "Automated model-based organ delineation for radiotherapy planning in prostatic region,"
Int. J. Radiation Oncology o Biology o Physics, vol.60, no.3, pp.973-980, 2004.

[119] M. Kaus, K.K. Brock, V. Pekar, L.A. Dawson, A.M. Nichol, and D.A. Jaffray, "Assessment of a model-based deformable image registration approach for radiation therapy planning," Int. J. Radiation Oncology o Biology o Physics, vol.68, no.2, pp.572-580, 2004.

[120] W. Li, S. Liao, Q. Feng, W. Chen, and D. Shen, "Learning image context for segmentation of the prostate in CT-guided radiotherapy," Physics in Medicine and Biology, vol.57, pp.1283-1308, 2012.

[121] G.J. Prince and C.J. Moore, "A method to calculate coverage probability from uncertainties in radiotherapy via a statistical shape model," Physics in Medicine and Biology, vol.52, pp.1947-1965, 2007.

[122] P. Shimari, B. Wu, R. Jacques, A. King, T. McNutt, R. Taylor, and M. Kazhdan, "A statistical approach for achievable dose querying in IMRT planning," Proc. 12th International Conference on Medical Image Computing and Computer Assisted Intervention (MICCAI 2009), Part III, Beijing, China, Lect. Notes Comput. Sci., vol.6363, pp.521-528, 2010.

[123] M. Kazhdan, P. Simari, T. McNutt, B. Wu, R. Jacques, M. Chung, and R. Taylor, "A shape relationship descriptor for radiation therapy planning," Proc. 12th International Conference on Medical Image Computing and Computer Assisted Intervention (MICCAI 2009), Part II, London, UK, Lect. Notes Comput. Sci., vol.5762, pp.100-108, 2009.

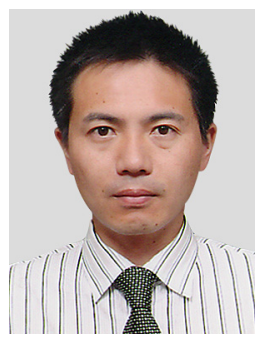

Ken'ichi Morooka received his M.S and $\mathrm{Ph} . \mathrm{D}$. degrees from the Graduate School of Information Science and Electrical Engineering, Kyushu University, in 1997 and 2000, respectively. He was associated with Institute of Systems \& Information Technologies/KYUSHU. From 2000 to 2006, he was Research Associate at Imaging Science and Engineering Laboratory, Tokyo Institute of Technology. From 2006 to 2010, he was Associate Professor in Digital Medicine Initiative, Kyushu University, Japan. Currently, he is Associate Professor in Graduate School of Information Science and Electrical Engineering, Kyushu University, Japan. His research interests cover medical imaging, computer graphics, and pattern recognition. 


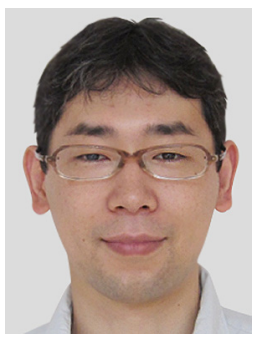

Masahiko Nakamoto received his B.S. M.S. and Ph.D degrees in Engineering from Osaka University, Japan in 1997, 1999, and 2002 respectively. From 2002 to 2004 , he was a Research Fellow at the Division of Functional Diagnostic Imaging, Osaka University Graduate School of Medicine. From 2004 to 2005, he was a Research Fellow at the Department of Disaster and Emergency Medicine, Graduate School of Medical Science, Kyushu University. In 2005, he joined the Division of Image Analysis of Osaka University Graduate School of Medicine as a faculty member. Since 2010, he is a Visiting Assistant Professor at the USC Institute of Urology, University of Southern California. He is currently an Assistant Professor at Osaka University Graduate School of Medicine and an Adjunct Assistant Professor of Graduate School of Information Science and Technology. His research fields include computer assisted surgery and medical image analysis.

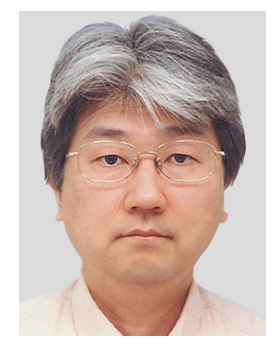

Yoshinobu Sato received his B.S., M.S. and $\mathrm{Ph} . \mathrm{D}$ degrees in Information and Computer Sciences from Osaka University, Japan in 1982 , 1984, 1988 respectively. From 1988 to 1992, he was a Research Engineer at the NTT Human Interface Laboratories. In 1992, he joined the Division of Functional Diagnostic Imaging of Osaka University Medical School as a faculty member. From 1996 to 1997, he was a Research Fellow in the Surgical Planning Laboratory, Harvard Medical School and Brigham and Women's Hospital. He is currently an Associate Professor at Osaka University Graduate School of Medicine and an Adjunct Associate Professor of MEI Center and Graduate School of Information Science and Technology, Osaka University. His research fields include medical image analysis, computer assisted surgery, and computational anatomy. Dr. Sato is a member of IEEE, MICCAI, and CAOS-International, and an editorial board member of Medical Image Analysis journal and the International Journal of Computer Assisted Radiology and Surgery. 